\title{
FATORES ASSOCIADOS AO BAIXO PESO AO NASCER EM SOBRAL-CE
}

FACTORS ASSOCIATED WITH LOW BIRTH WEIGHT IN SOBRAL, CEARÁ, BRAZIL

FACTORES ASOCIADOS AL BAJO PESO AL NACER EN SOBRAL, CEARÁ, BRASIL

Regina Célia Carvalho da Silva ${ }^{1}$

Márcia Maria Tavares Machado ${ }^{2}$

Carlos Henrique Alencar ${ }^{3}$

Ana Cristina Lindsay ${ }^{4}$

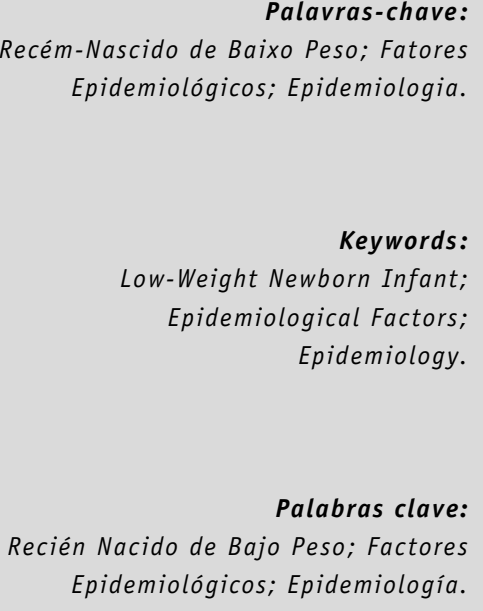

Submetido: 04 de Nov. de 2020

Aprovado: 14 de Dez. de 2020

Autor(a) para Correspondência: Regina Célia Carvalho da Silva

R. Anaid Andrade, 373 Centro - Sobral, CE CEP:62011-200 E-mail:reginacarvalho742@gmail.com

\section{RESUMO}

Este estudo teve por objetivo identificar os fatores associados ao baixo peso ao nascer (BPN) em Sobral-CE. Trata-se de estudo transversal com investigação de 2.181 nascimentos ocorridos entre 2006 e 2017, nos quais o desfecho foi o BPN e as variáveis explicativas foram classificadas segundo modelo teórico hierarquizado. A análise recorreu a Regressão de Poisson com variância robusta. O BPN foi mais frequente entre mulheres: a) não brancas; b) que tiveram até 9 anos de estudo; c) que ganhavam até 1 salário mínimo; e d) que não residiam com o companheiro - valores do nível distal; e) menores de 19 anos; f) que realizaram menos de 6 consultas pré-natal; g) que fumaram; e h) que ingeriram álcool na gestação - valores do nível intermediário; bem como i) com intercorrências de hipertensão; j) com diabetes; e k) com sangramento transvaginal durante a gestação - valores do nível proximal. A análise multivariada demonstrou associação com o BPN entre mulheres: a) com hipertensão; b) com diabetes; c) com sangramento transvaginal; d) menores de 19 anos; e) que fumaram na gestação; f) não brancas; e g) que realizaram menos de 6 consultas pré-natal. Concluiu-se haver possibilidade de reduzir a prevalência de BPN com ações voltadas $a$ : a) busca ativa precoce de gestantes para o pré-natal; b) combate ao fumo na gestação; e c) redução da gravidez na adolescência.

1. Enfermeira. Aluna de Doutorado em Saúde Pública na Universidade Federal do Ceará (UFC). E-mail: reginacarvalho742@gmail.com 0RCID: https://orcid.org/0000-0002-6124-8427

2. Professora na UFC. E-mail: marciamachadoufc@gmail.com 0RCID: https://orcid.org/0000-0002-0149-5792

3. Professor na UFC. E-mail: carlos.alencar@gmail.com ORCID: https://orcid.org/0000-0003-2967-532X

4. Professora na Harvard University. E-mail: ana.lindsay@umb.edu ORCID: https://orcid.org/0000-0002-2520$\underline{0493}$

Certificação de redação científica: E.L.Freire Editora. Edição de texto: Evandro L. Freire. Revisão de provas: Texto definitivo lido e validado pelos autoras. 


\section{ABSTRACT}

This study aimed to identify the factors associated with low birth weight (LBW) in Sobral, Ceará, Brazil. This is a cross-sectional study with an investigation of 2,181 births that occurred between 2006 and 2017, in which the outcome was LBW and explanatory variables were classified according to a hierarchical theoretical model. The analysis used Poisson Regression with robust variance. LBW was more frequent among women: a) who were nonwhite; b) who had up to 9 years of formal study; c) who earned up to 1 minimum wage; and d) who did not live with their partners - values at the distal level; e) under 19 years of age; f) who attended less than 6 prenatal appointments; g) who smoked; and h) who consumed alcohol during pregnancy - intermediate level values; as well as i) with complications of hypertension; $j$ ) with diabetes; and k) with transvaginal bleeding during pregnancy - values at the proximal level. Multivariate analysis showed an association with LBW among women: a) with hypertension; b) with diabetes; c) with transvaginal bleeding; d) under 19 years of age; e) who smoked during pregnancy; f) non-white; and $g$ ) who attended less than 6 prenatal appointments. It was concluded that there is a possibility to reduce the prevalence of $L B W$ through actions aimed at: a) early active search of pregnant women for prenatal care; b) fighting smoking during pregnancy; and c) reduction of teenage pregnancy.

\section{RESUMEN}

Este estudio tuvo como objetivo identificar los factores asociados con bajo peso al nacer (BPN) en Sobral, Ceará, Brasil. Se trata de un estudio transversal con una investigación de 2.181 nacimientos ocurridos entre 2006 y 2017 , en los que el resultado fue BPN y las variables explicativas se clasificaron según un modelo teórico jerárquico. El análisis utilizó Regresión de Poisson con varianza robusta. El BPN fue más frecuente entre mujeres: a) que no eran blancas; b) que tenían hasta 9 años de estudios formales; c) que ganaban hasta 1 salario mínimo; y d) que no convivian con sus parejas - valores a nivel distal; e) menores de 19 años; f) que asistieron a menos de 6 consultas prenatales; $g$ ) que fumaban; y h) que consumieron alcohol durante el embarazo - valores de nivel intermedio; así como i) con complicaciones de hipertensión; j) con diabetes; y $k$ ) con sangrado transvaginal durante el embarazo - valores a nivel proximal. El análisis multivariado mostró una asociación con el BPN entre las mujeres: a) con hipertensión; b) con diabetes; c) con sangrado transvaginal; d) menores de 19 años; e) que fumaron durante el embarazo; f) no blancas; y g) que asistieron a menos de 6 consultas prenatales. Se concluyó que existe la posibilidad de reducir la prevalencia de BPN a través de acciones dirigidas a: a) búsqueda activa temprana de gestantes para atención prenatal; b) lucha contra el tabaquismo durante el embarazo; y c) reducción del embarazo en la adolescencia.

\section{INTRODUÇÃO}

0 peso ao nascer é definido como a primeira medida do feto ou recém-nascido obtida após o nascimento e representa um importante parâmetro para a avaliação da saúde do recém-nascido. Trata-se de indicador das condições de saúde e nutricionais da mãe e do recém-nascido e constitui um alerta para o risco de morbimortalidade infantil ${ }^{1,2}$.

0 baixo peso ao nascer (BPN) corresponde aos recém-nascidos com peso abaixo de $2.500 \mathrm{~g}$, independentemente da idade gestacional, razão pela qual não se faz necessário o uso de uma curva de referência. 0 BPN é amplamente adotado em razão de sua simplicidade e de sua natureza objetiva; contudo, mostra desvantagens por limitar o peso a um valor isolado, não levando em consideração outros aspectos do desenvolvimento fetal. Variações do indicador baixo peso são identificadas na literatura, podendo levar a equívocos: a) baixo peso ao nascer
(BPN); b) pequeno para idade gestacional (PIG); c) restrição do crescimento intrauterino (RCIU); e d) restrição de crescimento fetal $(R C F)^{3}$.

A prevalência mundial do BPN é de $15,5 \%$, sendo sua distribuição desigual entre as regiões do mundo, com valores mais elevados nas subdesenvolvidas e menos elevados nas desenvolvidas ${ }^{4}$. Em 2012, o BPN foi estimado em $8,5 \%$ de todos os nascimentos no Brasil, com pequenas variações por região, destacando-se $9,2 \%$ no Sudeste e de $12 \%$ a $7,5 \%$ no Norte. 0 fato das taxas de BPN serem subestimadas nas regiões em desenvolvimento pode decorrer do grande número de partos domiciliares, nos quais o recém-nascido não é pesado, e ainda se constata deficiência quanto às informações disponibilizadas ${ }^{5}$.

0 peso ao nascer, o crescimento fetal e a duração da gestação podem estar associados ao recémnascido, à mãe ou mesmo a fatores ambientais. 0s principais fatores de risco relativos ao BPN são: a) 
idade materna; b) gestações de risco (especialmente com a ocorrência de hipertensão e diabetes); c) gestações múltiplas; d) número de filhos nascidos; e) sexo do recém-nascido; f) acesso às consultas pré-natal; g) peso materno prévio à gestação; h) tabagismo; i) consumo de álcool na gestação; j) parto induzido; e k) exposição a agrotóxicos ${ }^{6}$. Fatores socioeconômicos e ambientais, quando associados aos fatores maternos, podem modificar os resultados 4 . Vale ressaltar que os fatores de risco modificáveis são mais bem compreendidos para que se possa traçar estratégias de prevenção do BPN.

Embora se tenha como certeza que o BPN apresenta associação multifatorial, há evidências de que muitos fatores de risco se sobrepõem e que alguns estão mais presentes em determinadas regiões. Dessa forma, o objetivo deste estudo foi:

- Identificar os fatores associados ao baixo peso entre nascidos vivos de gestações únicas de mães residentes no Município de Sobral, no período entre 2006 e 2017.

\section{METODOLOGIA}

Trata-se de estudo transversal com uso do banco de dados da Estratégia Trevo de Quatro Folhas, realizado em Sobral, no período de 2006 a 2017. 0s dados para análise totalizaram 30.385 nascimentos, com informações registradas das mães e dos recémnascidos.

A Estratégia Trevo de Quatro Folhas foi criada em 2001, sendo ligada a e mantida pela Secretaria da Saúde do Município de Sobral, e é garantida por uma lei municipal que a institui como política pública municipal permanente. Foi implantada com o objetivo de reduzir a mortalidade infantil e atua em 4 frentes: a) atenção pré-natal de qualidade, com busca ativa e monitoramento de gestantes de risco; b) garantia de acesso a assistência hospitalar na ocasião do parto; c) vigilância do puerpério; e d) acompanhamento dos 2 primeiros anos de vida ${ }^{7}$.

0 banco de dados é composto por variáveis resultantes de um formulário com informações maternas e dados dos recém-nascidos. As informações maternas foram colhidas por um profissional da Secretaria da Saúde mediante entrevista presencial, com preenchimento de formulário estruturado, e também se coletavam dados do recém-nascido do prontuário hospitalar. Foram excluídos do estudo os dados dos partos gemelares, em razão desse tipo de gestação constituir importante preditor para baixo peso, o que poderia influenciar os resultados, e as unidades de observação com ausência de registro de variáveis que prejudicassem a análise. Foram excluídos, ainda, os nascidos vivos com peso abaixo de $500 \mathrm{~g}$, pois se evidenciou que tinham relação com prematuridade extrema.

De 2006 a 2017, o banco de dados continha 30.978 nascimentos. Destes foram excluídos 546 nascimentos de partos gemelares, 16 recém-nascidos sem definição de sexo e 31 nascimentos com peso abaixo de $500 \mathrm{~g}$, resultando em 30.385 unidades de observação para o estudo.

0 desfecho BPN $(<2.500 \mathrm{~g})$ foi contrastado com o não BPN (> $2.500 \mathrm{~g})$. As variáveis explicativas foram categorizadas e classificadas segundo modelo teórico hierarquizado em 3 níveis: a) nível distal - características sociodemográficas (cor da pele, escolaridade, renda, situação civil, área de residência); b) nível intermediário - características maternas (idade da mãe, gestação desejada, tipo de parto, número de consultas pré-natal, primeira consulta pré-natal, fumo durante a gestação, uso de bebidas alcoólicas durante a gestação); e c) nível proximal - caraterísticas obstétricas (intercorrências na gestação, hipertensão, diabetes, infecção urinária, infecção vaginal, sangramento transvaginal).

As variáveis foram categorizadas em dicotômicas e aplicou-se o teste do qui-quadrado de Pearson para avaliar a significância estatística. $\mathrm{Na}$ análise bivariada se empregou o cálculo da razão de prevalência bruta com as variáveis com valor $p$ abaixo de 0,020 . Na análise multivariada, para verificar a associação entre o desfecho e as variáveis explicativas, usouse a Regressão de Poisson com variância robusta para estimar a razão de prevalência ajustada, sendo elegiveis as variáveis com nível de significância abaixo de $10 \%$. A entrada das variáveis explicativas na modelagem ocorreu passo a passo, conforme o nível hierárquico. Para a permanência da variável no modelo, adotou-se o nível de significância de $5 \%$ e 0

\section{...há evidências de que muitos fatores de risco se sobrepõem...}


intervalo de confiança de $95 \%$. Para as análises, recorreu-se a o software STATA, versão 13.0.

0 estudo foi aprovado pelo Comitê de Ética em Pesquisa da Universidade Estadual Vale do Acaraú (CEP/UVA), sob o Parecer n. 4.016.736/2020.

\section{RESULTADOS}

Dentre os 30.385 nascimentos ocorridos de 2006 a 2017 elegíveis para o estudo, 2.181 (7,18\%) nasceram com peso inferior a $2.500 \mathrm{~g}$. Houve um aumento de 1,5\% nas taxas de BPN em gestações únicas de 2006 a 2017.

$\mathrm{Na}$ análise das características sociodemográficas, que representa o nível mais distal no modelo hierárquico, o BPN foi mais frequente nas mulheres não brancas $(7,43 \%)$, nas que tiveram até 9 anos de estudo (7,64\%), nas que ganhavam até 1 salário mínimo (7,63\%) e naquelas que não residiam com o companheiro $(8,23 \%)$, como ilustra a Tabela 1.

Tabela 1 - Distribuição do peso ao nascer segundo características sociodemográficas de mulheres residentes em Sobral-CE (2006-2017)

\begin{tabular}{|c|c|c|c|}
\hline Nivel distal & & BPN & \\
\hline Variável & $\begin{array}{c}\text { Não } \\
\text { N (\%) }\end{array}$ & $\begin{array}{c}\text { Sim } \\
\text { N (\%) }\end{array}$ & $P$ \\
\hline Cor da pele & & & 0,000 \\
\hline Branca & $3.497(96,6)$ & $197(3,4)$ & \\
\hline Não branca & $24.751(92,57)$ & $1.986(7,43)$ & \\
\hline Escolaridade & & & 0,023 \\
\hline Até 9 anos de estudo & $10.237(92,36)$ & $847(7,64)$ & \\
\hline Acima de 9 anos de estudo & $17.964(93,08)$ & $1.336(6,92)$ & \\
\hline Renda & & & 0,008 \\
\hline Menos de 1 salário mínimo & $13.925(92,37)$ & $1.150(7,63)$ & \\
\hline De 1 a 5 salário(s) mínimo(s) & $13.782(93,20)$ & $1.005(6,80)$ & \\
\hline 6 ou mais salários mínimos & $497(94,31)$ & $30(5,69)$ & \\
\hline Estado civil & & & 0,000 \\
\hline Reside com o companheiro & $22.369(93,08)$ & $1.662(6,92)$ & \\
\hline Não reside com o companheiro & $5.835(91,77)$ & $523(8,23)$ & \\
\hline
\end{tabular}

Fonte: Elaborada pelos autores.

A Tabela 2 apresenta o nível intermediário relacionado às características maternas. Considerando tais características, o baixo peso foi mais frequente nas mulheres com menos de 19 anos (8,90\%), nas que tiveram menos de 6 consultas pré-natal $(13,44 \%)$, nas que a primeira consulta ocorreu no terceiro trimestre $(10,09 \%)$, nas que fumaram $(11,69 \%)$ e naquelas que ingeriram álcool $(12,19 \%)$ na gestação.

Tabela 2 - Distribuição do peso ao nascer segundo características maternas de mulheres residentes em SobralCE (2006-2017)

\begin{tabular}{lccc}
\hline Nivel distal & & BPN \\
\hline Variável & Não & Sim & N $(\%)$ \\
\hline Idade materna & N $\%)$ & & 0,000 \\
$\leq 19$ anos & & $554(8,90)$ & \\
20 a 34 anos & $5,672(91,10)$ & $1.402(6,53)$ \\
$\geq 35$ anos & $20,070(93,47)$ & $229(8,51)$ \\
\hline
\end{tabular}




\begin{tabular}{|c|c|c|c|}
\hline Nivel distal & & BPN & \\
\hline Variável & $\begin{array}{c}\text { Não } \\
\text { N (\%) }\end{array}$ & $\begin{array}{c}\text { Sim } \\
\text { N (\%) }\end{array}$ & $P$ \\
\hline Consulta pré-natal & & & 0,000 \\
\hline Até 5 consultas & $5.161(86,56)$ & $801(13,44)$ & \\
\hline 6 ou mais consultas & $23.036(94,33)$ & $1.384(5,67)$ & \\
\hline Primeira consulta pré-natal & & & 0,006 \\
\hline $1^{\circ}$ trimestre & $19.056(93,15)$ & $1.402(6,85)$ & \\
\hline $2^{\circ}$ trimestre & $4.626(92,35)$ & $423(7,65)$ & \\
\hline $3^{\circ}$ trimestre & $392(89,91)$ & $44(10,09)$ & \\
\hline Fumou na gestação & & & 0,000 \\
\hline Sim & $1.005(88,31)$ & $133(11,69)$ & \\
\hline Não & $27.199(92,48)$ & $2.052(7,52)$ & \\
\hline Ingeriu álcool na gestação & & & 0,000 \\
\hline Sim & $353(87,81)$ & $49(12,19)$ & \\
\hline Não & $27.736(92,88)$ & $2.136(7,12)$ & \\
\hline
\end{tabular}

Fonte: Elaborada pelos autores.

No nível proximal relacionado com as características obstétricas, os recém-nascidos com baixo peso foram mais frequentes entre as mulheres hipertensas $(14,34 \%)$, as diabéticas $(12,20 \%)$ e as que apresentaram sangramento transvaginal $(15,92 \%)$ durante a gestação. Nas mulheres com infecção urinária tratada $(6,07 \%)$, bem como naquelas com infecção vaginal tratada $(5,68 \%)$ se verificou frequência menor de BPN em comparação àquelas que não tiveram ou não foram identificadas com as infecções mencionadas (Tabela 3 ).

Tabela 3 - Distribuição do peso ao nascer segundo características obstétricas de mulheres residentes em Sobral-CE (2006-2017)

\begin{tabular}{|c|c|c|c|}
\hline Nivel distal & & BPN & \\
\hline Variável & $\begin{array}{c}\text { Não } \\
\text { N (\%) }\end{array}$ & $\begin{array}{c}\text { Sim } \\
\text { N (\%) }\end{array}$ & $P$ \\
\hline Hipertensão & & & 0,000 \\
\hline Sim & $466(85,66)$ & $78(14,34)$ & \\
\hline Não & $27.738(92,94)$ & $2.107(7,06)$ & \\
\hline Diabetes & & & 0,000 \\
\hline Sim & $540(87,80)$ & $75(12,20)$ & \\
\hline Não & $27.664(92,91)$ & $2.110(7,09)$ & \\
\hline Sangramento transvaginal & & & 0,000 \\
\hline Sim & $597(84,08)$ & $113(15,92)$ & \\
\hline Não & $27.607(92,91)$ & $2.072(6,92)$ & \\
\hline Infecção urinária tratada & & & 0,000 \\
\hline Sim & $8.350(93,93)$ & $540(6,07)$ & \\
\hline Não & $19.854(92,14)$ & $1.645(7,86)$ & \\
\hline Infecção vaginal tratada & & & 0,000 \\
\hline Sim & $7.155(94,32)$ & $431(5,68)$ & \\
\hline Não & $21.049(92,31)$ & $1.754(7,69)$ & \\
\hline
\end{tabular}

Fonte: Elaborada pelos autores. 
Na análise bivariada, as variáveis gravidez desejada e tipo de parto não apresentaram significância estatística, não sendo, portanto, selecionadas para a análise multivariada. Foram escolhidas para as categorias de referência as variáveis que apresentassem o menor percentual de frequência de BPN.

$\mathrm{Na}$ Tabela 4 se verificam as razões de prevalência brutas ( $R P b$ ) e as razões de prevalência ajustadas ( $R P a$ ) pelo modelo de Regressão de Poisson com variância robusta e os respectivos intervalos de confiança de $95 \%$ para o desfecho BPN. No modelo final ficaram apenas as variáveis com nível de significância abaixo de $5 \%$.

Tabela 4 - Razões de prevalência do baixo peso ao nascer brutas e ajustadas de mulheres residentes em SobralCE (2006-2017)

\begin{tabular}{llllll}
\hline & RPb & IC (95\%) & RPa & IC (95\%) & $P$ \\
\hline Hipertensão & 2.03 & $1.65-2.50$ & 2.10 & $1.63-2.69$ & 0,00 \\
Diabetes & 1.72 & $1.38-2.13$ & 1.65 & $1.28-2.12$ & 0,00 \\
Sangramento transvaginal & 2.27 & $1.91-2.71$ & 2.35 & $1.99-2.79$ & 0,00 \\
Fumou na gestação & 1.66 & $1.41-1.96$ & 1.69 & $1.40-2.04$ & 0,00 \\
Consultas pré-natal $(<6)$ & 2.36 & $2.17-2.56$ & 2.60 & $2.36-2.85$ & 0,00 \\
Idade (<19 anos) & 1.36 & $1.24-1.49$ & 1.18 & $1.11-1.26$ & 0,00 \\
Cor da pele (não branca) & 1.37 & $1.19-1.58$ & 1.52 & $1.31-1.77$ & 0,00 \\
\hline
\end{tabular}

Fonte: Elaborada pelos autores.

Segundo a análise multivariada, apresentaram associação com BPN as mulheres que tiveram hipertensão (RP 2.10, IC 1.63-2.69), diabetes (RP 1.65, IC 1.28-2.12) e sangramento transvaginal (RP 2.35, IC 1.99-2.79) na gestação, bem como as mulheres com menos de 19 anos (RP 1.18, IC 1.11-1.26), as que fumaram na gestação (RP 1.69, IC 1.40-2.04), as não brancas (RP 1.52, IC 1.31-1.77) e aquelas que tiveram menos de 6 consultas pré-natal (RP 2.36, IC 2.36-2.85).

\section{DISCUSSÃO}

0 peso ao nascer, junto com a estatura e o perímetro cefálico, são medidas importantes que constituem parâmetros iniciais para o acompanhamento do crescimento e desenvolvimento do recém-nascido. Quando associado à idade gestacional, o peso a nascer compõe um relevante índice de avaliação do crescimento fetal e de padrão de cuidado para os recém-nascidos de risco.

Os fatores associados ao BPN no Município de Sobral são mais evidentes entre as mulheres que apresentaram intercorrências na gestação, como: a) hipertensão; b) diabetes; e c) sangramento transvaginal. Ademais, evidenciou-se que as manifestações fisiológicas ou patológicas durante a gravidez geralmente estão associadas a risco de prematuridade e, consequentemente, ao BPN. Dentre elas, encontram-se: a) sangramento transvaginal; b) síndromes hipertensivas; c) diabetes gestacional; d) depressão; e) estresse; f) doenças crônicas; e g) infecções ${ }^{8}$. Neste estudo, optou-se por investigar o baixo peso isoladamente, sem considerar a idade gestacional.

A faixa etária abaixo de 19 anos apresentou associação com o BPN. Os estudos apontam ser mais comum observar prematuridade entre mães menores de 18 anos. A associação pode ser explicada pelo baixo peso dos recém-nascidos prematuros. Esses achados foram evidenciados, inclusive, em países desenvolvidos ${ }^{9-11}$.

Dentre as características sociodemográficas, apenas a variável cor da pele permaneceu no modelo final. Dados apontam influxos da desigualdade relativa a cor/raça que se estendem por todo o processo gravidez/ parto e destaca-se que essa variável recebe intensa influência de outras, como: a) renda; b) escolaridade; e c) acesso aos serviços de saúde ${ }^{12}$.

Verificou-se que o ato da mulher fumar durante a gestação teve associação com BPN. Vários estudos expressaram o uso de tabaco durante a gravidez, bem como a exposição da gestante ao ambiente com resíduo de tabaco como fatores a considerar para risco de BPN ${ }^{8,13-15}$. Existem mais de 3.000 produtos químicos no tabaco e seus efeitos biológicos não são totalmente conhecidos. Sabe-se que a nicotina e o monóxido de carbono são poderosos vasoconstrictores que se associam a danos placentários e à diminuição do fluxo sanguíneo uteroplacentário, o que pode levar à restrição do crescimento intrauterino e à indução de parto prematuro ${ }^{16}$. 
Um estudo realizado no Reino Unido verificou que houve aumento progressivo do peso médio dos recémnascidos de 1996 a 2012. Dentre outras causas, os autores atribuem tal achado às políticas públicas para redução do fumo e à legislação que proíbe o fumo em lugares públicos, introduzida no país em $2007^{17}$.

No Brasil, o Ministério da Saúde recomenda pelo menos 6 consultas pré-natal, que devem ter início no primeiro trimestre de gravidez. 0 número de consultas pré-natais esteve fortemente associado ao BPN neste estudo. A assistência pré-natal adequada deve ocorrer por meio de: a) busca ativa precoce da gestante (antes da $12^{\mathrm{a}}$ semana de gravidez); b) garantia de, no mínimo, 6 consultas, com condutas acolhedoras e humanizadas; $c$ ) realização de exames; d) detecção de e intervenção precoce em situações de risco; e e) plano de vinculação da gestante à maternidade, assegurando acesso aos serviços de saúde desde o atendimento básico até o cuidado hospitalar de alto risco ${ }^{18}$.

Os resultados de um estudo no Brasil mostraram que a cobertura da assistência pré-natal é elevada no país, no entanto, a qualidade dessa assistência é baixa. Constatou-se que $60,6 \%$ das mulheres iniciaram o pré-natal até a $12^{\text {a }}$ semana de gravidez e $73,1 \%$ alcançaram o número mínimo de consultas pré-natal previstas até o momento do parto ${ }^{19}$.

Uma metanálise apresentou dados sobre a associação do peso ao nascer com o estado civil da mãe ${ }^{20}$. Mães solteiras estavam associadas ao aumento do risco de BPN, o que não foi evidenciado neste estudo.

Recém-nascidos em condições de baixo peso, quando comparados àqueles com peso igual ou superior a $2.500 \mathrm{~g}$, mostram-se mais propensos a apresentar: a) prejuízos no neurodesenvolvimento; b) problemas de desempenho escolar; e c) dificuldades comportamentais ${ }^{21,22}$. Ressaltase, porém, que as condições socioeconômicas da família e a disponibilidade dos serviços de saúde, especialmente do seguimento ambulatorial por equipe multiprofissional, atenuam ou agravam de modo significativo 0 desencadeamento de tais dificuldades ${ }^{23}$.

Portanto, o futuro desenvolvimento da criança se encontra intimamente associado à saúde do recémnascido, assim como às características maternas, às variáveis do ambiente familiar e aos programas de intervenção precoce para detecção e reversão de problemas ${ }^{23}$. A intervenção precoce atenua a

\section{...o futuro \\ desenvolvimento da \\ criança se encontra \\ intimamente associado \\ à saúde do recém \\ nascido...}

tendência negativa das crianças com baixo peso em seus primeiros anos de vida, algo que pode estenderse até a adolescência.

\section{CONCLUSÃO}

Neste estudo, concluiu-se haver possibilidade de reduzir a prevalência de BPN com ações voltadas à busca ativa precoce de gestantes para o pré-natal, ao combate ao fumo na gestação e à redução da gravidez na adolescência. Já a intervenção em intercorrências na gestação, como hipertensão, diabetes e sangramento transvaginal, mostra-se difícil porque, muitas vezes, associa-se ao estado de saúde prévio ou alguma alteração biológica apresentada pela gestante. No entanto, tais intercorrências devem ser consideradas manifestações de alerta, mesmo que a gestação ainda não seja considerada de risco.

\section{CONTRIBUIÇÃO DOS AUTORES}

Regina Célia Carvalho da Silva contribuiu com a realização da pesquisa e a redação do manuscrito. Márcia Maria Tavares Machado e Ana Cristina Lindsay contribuíram com a revisão crítica do manuscrito. Carlos Henrique Alencar contribuiu com a realização da pesquisa e o delineamento do estudo.

\section{REFERÊNCIAS}

1. Lawn JE, Gravett MG, Nunes TM, Rubens CE, Stanton C, Group GR. Global report on preterm birth and stillbirth: Definitions, description of the burden and opportunities to improve data. BMC Pregnancy and Childbirth. $2010 ; 10(1):$ S1.

2. World Health Organization. Global nutrition targets 2025: low birth weight policy brief. Geneva: WHO; 2014.

3. Kramer MS. Born too small or too soon. The Lancet Global Health. 2013:1(1):7-8. 
4. Demelash H, Motbainor A, Nigatu D, Gashaw K, Melese A. Risk factors for low birth weight in Bale zone hospitals, South-East Ethiopia: a case-control study. BMC Pregnancy \& Childbirth. 2015;(15):264.

5. Mendes CQS, Cacella BCA, Mandetta MA, Balieiro MMFG. Baixo peso ao nascer em município da região sudeste do Brasil. Rev Bras Enferm [serial on the internet]. 2015 [cited 2020 Dec 14];68(6):857-63. Available from: https://www.scielo.br/pdf/reben/ v68n6/0034-7167-reben-68-06-1169.pdf

6. Capelli JCS, Rocha CMM, Monteiro LS, Sperandio $\mathrm{N}$, Anastácio AS, Pereira $S$, et al. Baixo peso ao nascer e fatores associados ao pré-natal: um estudo seccional em uma maternidade referência de Macaé. Saúde Redes [serial on the internet]. 2020 [cited 2020 Dec 14];6(1):163-73. Available from: http:// revista.redeunida.org.br/ojs/index.php/rede-unida/ article/view/2342/495

7. Sousa FJS, Sucupira ACSL, Aguiar ISM, Mesquita VAL, Sales ENBG. Programa Trevo de Quatro Folhas: uma ação efetiva para a redução da mortalidade infantil em Sobral - Ceará. Sanare (Sobral, Online) [serial on the internet]. 2012 [cited 2020 Dec 14];11(1):60-5. Available from: https://sanare. emnuvens.com.br/sanare/article/view/268

8. Harrison MS, Goldenberg RL. Global burden of prematurity. Seminars in Fetal \& Neonatal Medicine. 2016;21:74-9.

9. Kenny LC, Lavender T, McNamee R, O'Neill SM, Mills T, Khashan AS. Advanced maternal age and adverse pregnancy outcome: evidence from a large contemporary cohort. PloS 0ne. 2013;8(2):e56583.

10. Blomberg M, Tyrberg RB, Kjølhede P. Impact of maternal age on obstetric and neonatal outcome with emphasis on primiparous adolescents and older women: a Swedish Medical Birth Register Study. BMJ Open. 2014;4(11):e005840.

11. Torchina H, Ancel Y. Prématurité spontanée épidémiologie et facteurs de risque de la prématurité. Journal de Gynécologie Obstétrique et Biologie de la Reproduction. $2016 ; 45(10): 1213-30$.

12. Leal MC, Gama SGN, Pereira APE, Pacheco VE, Carmo CN, Santos RV. A cor da dor: iniquidades raciais na atenção pré-natal e ao parto no Brasil. Cad Saúde Pública [serial on the internet]. 2017 [cited 2020 Dec 14];33(1):e00078816. Available from: https:// www.scielo.br/pdf/csp/v33s1/1678-4464-csp-33s1-e00078816.pdf

13. Dessì A, Corona L, Pintus R, Fanos V. Exposure to tobacco smoke and low birth weight: from epidemiology to metabolomics. Expert Rev Proteomics. $2018 ; 15(8): 647-56$.
14. Ko TS, Tsai LY, Chu LC, Yen SJ, Leung C, Chen $\mathrm{CY}$, et al. Parental smoking during pregnancy and its association with low birth weight, small for gestational age, and preterm birth offspring: a birth cohort study. Pediatrics \& Neonatology. $2014 ; 55(1): 20-7$.

15. Khattar D, Awasthi S, Das V. Residential environmental tobacco smoke exposure during pregnancy and low birth weight of neonates: case control study in a public hospital in Lucknow, India. Indian Pediatrics. 2013;50:134-8.

16. Goldenberg RL, Culhane JF, Iams JD, Romero R. Epidemiology and causes of preterm birth. Lancet. $2008 ;(371): 75-84$.

17. Ghosh RE, Berild JD, Sterrantino AF, Toledano MB, Hansell LA. Birth weight trends in England and Wales (1986-2012): babies are getting heavier. Arch Dis Child Fetal Neonatal. 2018;103(3):264-70.

18. Brasil. Atenção ao pré-natal de baixo risco. Brasília (DF): Ed. Ministério da Saúde; 2013.

19. Viellas EF, Domingues RMSM, Dias MCB, Gama SGN, Theme Filha MM, Costa JN, et al. Assistência pré-natal no Brasil. Cad Saúde Pública. 2014;30(Suppl):85-100.

20. Shan PS, Zao J, Ali S. Maternal marital status and birth outcomes: a systematic review and metaanalyses. Maternal Child Health J. 2011;15(7):1097109.

21. Mukhophadhyay K, Mahajan R, Malhi P, Kumar A. Neurodevelopmental outcome of extremely low birth weight children at corrected age of two years. Indian Pediatrics. 2016;53(5):391-3.

22. Hintz SR, Vohr BR, Bann CM, Taylor HG, Das $A$, Gustafson $K E$, et al. Preterm neuroimaging and school-age cognitive outcomes. Pediatrics. 2018;142(1):e20174058.

23. Rugolo LMSS. Importância da monitorização do desenvolvimento em recém-nascidos prematuros. Rev Paul Pediatr [serial on the internet]. 2012 [cited 2020 Dec 14];30(4):460-1. Available from: http://www.scielo.br/scielo.php?script $=$ sci arttext\&pid $=$ S0103-05822012000400001\&lng =en
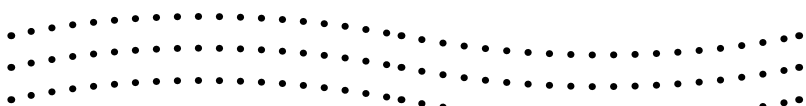